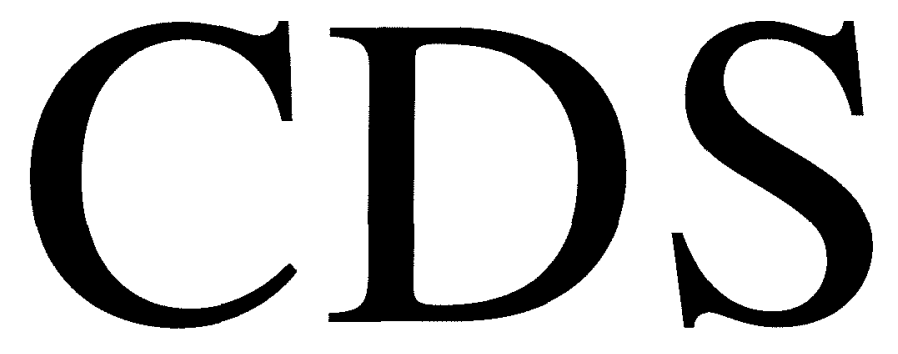

TECHNICAL MEMORANDUM NO. CIT-CDS 95-021

July, 1995

"Pattern Evocation and Geometric Phases in Mechanical Systems with Symmetry"

Jerrold E. Marsden and Jürgen Scheurle

\title{
Control and Dynamical Systems
} California Institute of Technology Pasadena, CA 91125 


\title{
Pattern Evocation and Geometric Phases in Mechanical Systems with Symmetry
}

\author{
Jerrold E. Marsden* \\ Control and Dynamical Systems \\ Caltech 104-44, Pasadena, CA 91125 \\ marsden@cds.caltech.edu \\ Jürgen Scheurle ${ }^{\dagger}$ \\ Institut für Angewandte Mathematik \\ Bundesstrasse 55, D-20146 Hamburg, Germany \\ scheurle@math.uni-hamburg.de \\ to appear in Dynamics and Stability of Systems
}

June, 1993; this version: July 31, 1995

\begin{abstract}
This paper is concerned with the relation between the dynamics of a given Hamiltonian system with a given symmetry group and its reduced dynamics. We illustrate the process of visualization of reduced orbits using the double spherical pendulum. In this process of visualization, one sees certain patterns when the dynamics is viewed relative to rotating frames with certain critical angular velocities. By using the reduced dynamics, we also explain these patterns.

We show that if the motion on the phase space reduced by a continuous symmetry group at a given momentum level is periodic, then there is a uniformly rotating frame, that is, a one parameter group motion, relative to which the unreduced trajectory is periodic with the same period. If the continuous symmetry group of the system is abelian, which corresponds to the system having cyclic variables, we derive an explicit expression for the required angular velocity in terms of the dynamic phase (an average of the mechanical connection) and the geometric phase (the holonomy of the mechanical connection). We show that one can also find such a frame if the reduced orbit is quasiperiodic and a KAM condition is satisfied. The almost periodic case is also discussed.

An important aspect of this procedure is how to use it in the presence of discrete symmetries. We show that, under appropriate conditions, the visualized orbit has, relative to a suitable uniformly rotating frame, the same temporal behavior and discrete symmetries as the reduced orbit. Since these spatio-temporal patterns are not apparent with respect to most frames, we call the phenomenon pattern evocation.
\end{abstract}

\footnotetext{
* Research partially supported by NSF, DOE, and NATO.

${ }^{\dagger}$ Research partially supported by DFG and NATO.
} 


\section{Contents}

1 Introduction 2

1.1 Visualization for the Double Spherical Pendulum . . . . . . . . . . . . . 2

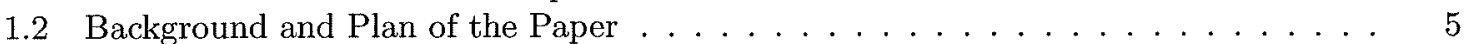

2 Systems with a General Continuous Symmetry Group 6

2.1 Existence of Uniformly Rotating Frames for Relatively Periodic Orbits . . . . . . . 7

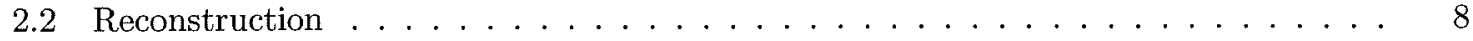

2.3 The Mechanical Connection . . . . . . . . . . . . . . . . . . . 10

3 Systems with Cyclic Coordinates 10

3.1 Notation . . . . . . . . . . . . . . . . . . . . . . . . 10

3.2 The Frame Angular Velocity Formula . . . . . . . . . . . . . . . . . 13

3.3 The Quasiperiodic Case . . . . . . . . . . . . . . . . . . . . . . 14

4 Discrete Symmetries $\quad 16$

4.1 Introduction . . . . . . . . . . . . . . . . . . . . . 16

4.2 The relation between quotient groups and semidirect products . . . . . . . . . . 17

4.3 Preservation properties of the momentum map . . . . . . . . . . . . . . 18

4.4 Relative Equilibria . . . . . . . . . . . . . . . . . . . . . . . . 19

4.5 General Time Dependent Orbits . . . . . . . . . . . . . . . . . . . . . . . 19

5 The Double Spherical Pendulum 20

5.1 The Basic Equations and their Continuous Symmetry . . . . . . . . . . . 20

5.2 The Discrete Symmetries . . . . . . . . . . . . . . . . . . . 22

\section{Introduction}

We will begin by explaining the phenomenon we wish to study in the case of the double spherical pendulum.

\subsection{Visualization for the Double Spherical Pendulum}

Consider the mechanical system consisting of two spherical pendula coupled with spherical joints and moving without friction in a gravitational field. (See Figure 1.1.1).

In this figure, the position vectors of each pendulum relative to their hinge points are denoted $\mathbf{q}_{1}$ and $\mathbf{q}_{2}$. These vectors are assumed to have fixed lengths $l_{1}$ and $l_{2}$ and the pendula masses are denoted $m_{1}$ and $m_{2}$.

The continuous symmetry group for the double spherical pendulum is the group of rotations around the $z$-axis, that is, the axis of gravity. By reduction relative to this group, we mean that a value of the angular momentum about the $z$-axis is fixed and configuration points related by rotations about the $z$-axis are identified. The full configuration space is parameterized by the positions of both bobs, and the reduced configurations are parameterized by $r_{1}, r_{2}, \theta$, where $r_{i}$ is the radial polar coordinate of the $i$ th pendulum bob, and where $\theta$ is the angle between the two vertical planes each of which contains one of the two bobs and the suspension point. (For details, see Marsden and Scheurle [1993a].)

A relative equilibrium for the double spherical pendulum is a motion of the pendulum bobs that is given by a uniform rotation of the bobs about the $z$-axis with a fixed angular velocity $\xi_{e}$. In such a case, the reduced orbit is a fixed point; that is, the variables $r_{1}, r_{2}, \theta$ are fixed in time. In this 


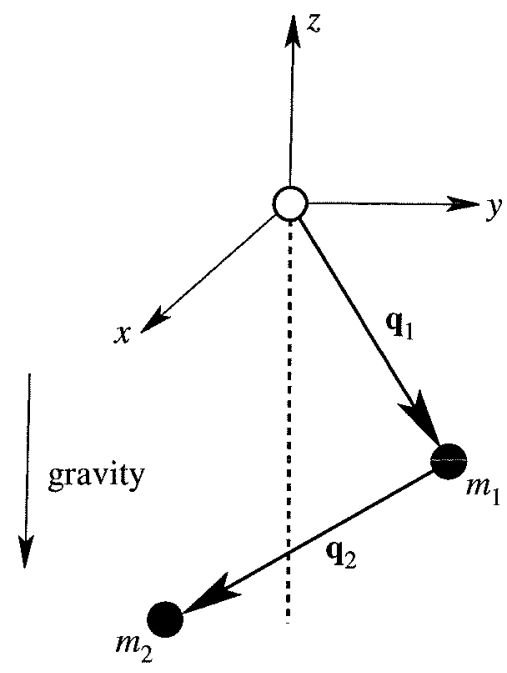

Figure 1.1.1: The configuration space for the double spherical pendulum consists of two copies of the two-sphere

simple case, if one views the bobs from a frame rotating with the same angular velocity $\xi_{e}$, the bobs will of course appear stationary.

A relatively periodic (resp. relatively quasiperiodic) orbit is one whose corresponding reduced orbit is periodic (resp. quasiperiodic). Concretely, this means that the variables $r_{1}, r_{2}, \theta$ will be periodic (resp. quasiperiodic) in time. For such orbits the visualization process is not so obvious as in the case of relative equilibria.

An interesting feature is that if one views a relative periodic orbit of the pendulum bobs, then from the frame of a rotating platform (or camera), rotating with a suitably chosen angular velocity (in the same general direction as the motion of the bobs), the actual positions of the pendulum bobs will appear periodic in time. As the frame of the platform is adjusted to "resonant" values, one also sees patterns emerge. We see similar pattern formations for more complicated orbits whose corresponding reduced orbits need not be periodic.

In the following three figures (from Marsden, Scheurle and Wendlandt [1995]), we show one orbit (one set of initial data) visualized at three different frame angular velocities, which are denoted by $\xi$. The upper curves in each figure show the actual trajectories of the bobs in three space, relative to the rotating frame. The lower two curves show the projections of these curves onto the horizontal plane. This projected information is enough to capture the full information about the system in view of the length constraints.

In figure 1.1.2 we show the orbit of the pendulum bobs relative to the lab frame (the frame angular velocity $\xi$ is zero). 


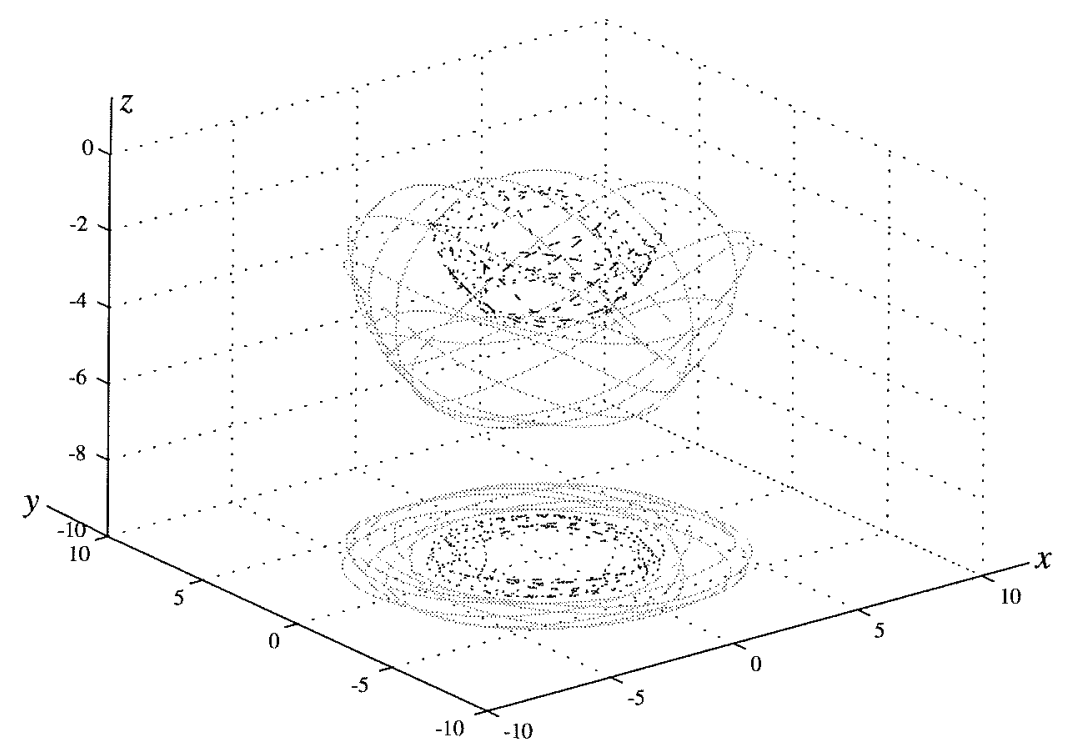

Figure 1.1.2: An orbit of the double spherical pendulum relative to the lab frame; that is, with $\xi=0$.

A pattern that emerges when the frame angular velocity is increased to the value $\xi=0.8550$ is shown in Figure 1.1.3.

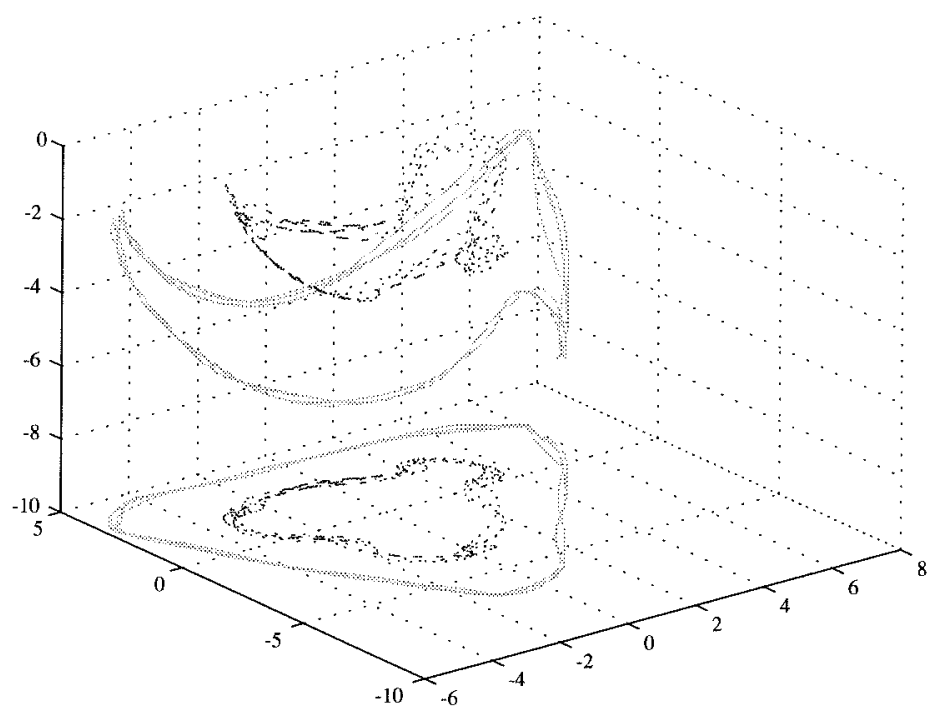

Figure 1.1.3: The same orbit of the double spherical pendulum at a resonant frame angular velocity.

The final figure 1.1.4 shows a particular critical value $\xi_{c}$ of the frame angular velocity at which the bobs do not encircle the $z$-axis. As we shall see later on, this special value captures the dynamics of the corresponding reduced orbit. In the body of the paper we will be developing formulae for the value of this critical velocity.

As we shall explain in detail later, the discrete symmetry of the double spherical pendulum given by reflection in a vertical plane together with reversibility gives rise to a discrete $\mathbb{Z}_{2}$ symmetry of the reduced system. Thus, reduced trajectories can have a spatio-temporal symmetry associated with this discrete symmetry. As we show later and as the figures indicate, such symmetries are inherited 


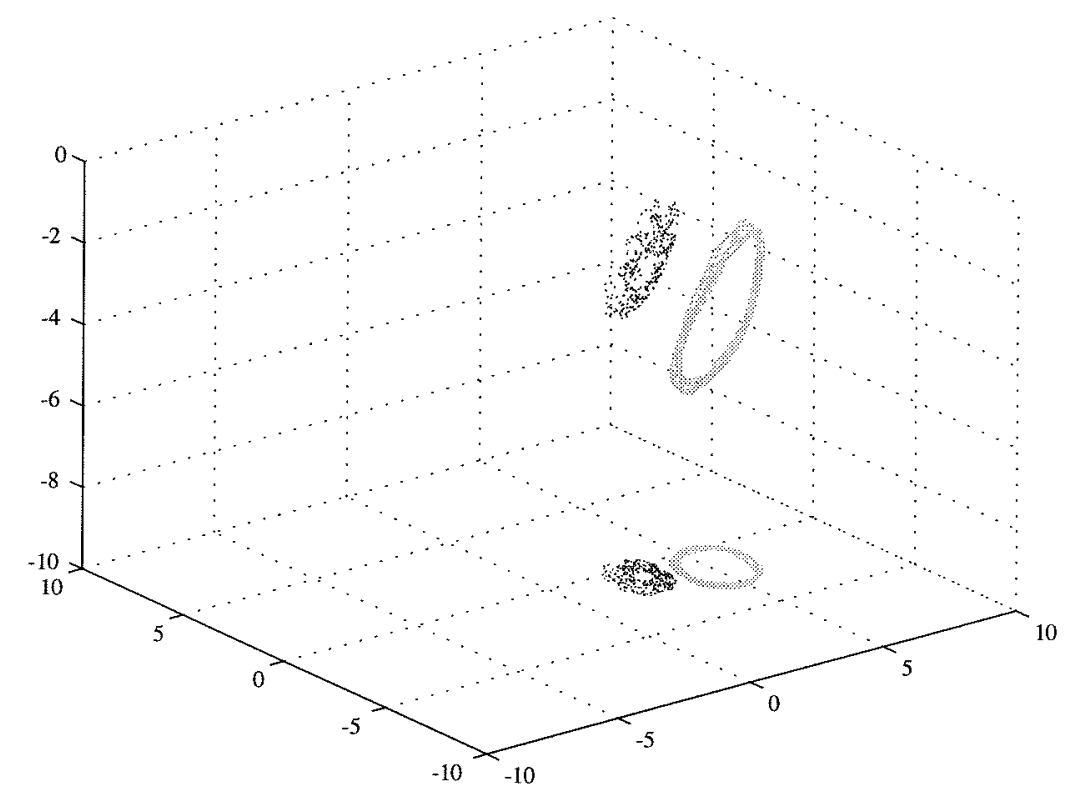

Figure 1.1.4: The phase portrait of the double spherical pendulum at the critical frame angular velocity.

by the trajectories relative to the rotating frame.

We notice here, and will discuss in greater detail in Marsden Scheurle and Wendlandt [1995], the following formula applies when the reduced orbit is periodic (but actually holds surprisingly well in other cases too):

$$
\xi_{m}-\xi_{c}=\frac{n}{m} F
$$

Here, $\xi_{m}$ is the frame angular velocity at which one sees an $m$-fold symmetric pattern, $F$ is the frequency of the reduced periodic orbit, and $n$ is an integer.

\subsection{Background and Plan of the Paper}

The phenomena of visualization of orbits in the reduced space was studied, especially for the case of the dynamics of point vortices, in Kunin, Hussain, Zhou and Prishepionok [1992]. They observed phenomenon related to what we described above numerically for the case of four point vortices. The motion of the vortices is observed to simplify in the sense of developed patterns relative to an appropriately chosen rotating frame (but the angular velocity of the frame might not be constant).

The present paper puts this phenomenon into the general context of mechanical systems with symmetry and uses, in particular, averaging, reconstruction, and the theory of dynamic and geometric phases. (See Newton [1994] for the use of geometric phases in problems of point vortices and for further references.) One of our main points is that for motions whose reduced trajectory is periodic (resp. quasiperiodic with a KAM condition holding), the frame angular velocity relative to which the orbit itself is periodic (resp. quasiperiodic) with the same frequency can be chosen to be constant. The value of the constant angular velocity involves a combination of the geometric and dynamic phase, as will be demonstrated.

As we shall see, it is not sufficient to choose the so-called Tisserand frame, which is the frame that rotates with an angular velocity chosen so that the instantaneous (or locked) moment of inertia tensor times the angular velocity equals the angular momentum; (this was introduced in Chapter 30 of Tisserand's 1890 book on celestial mechanics). The Tisserand frame need not be uniformly rotating; however, its average also does not accomplish the desired task, even for cyclic variables 
and relative periodic orbits, since it fails to take into account the geometric phase. On a historical note we mention that many of the ideas herein were also relevant to Poincaré and it would be of interest to have a closer look at his work from this perspective.

The plan of the paper is to first give some general results on the existence of uniformly rotating frames valid for any symmetry group. Following this we discuss symmetries that drop to the reduced space and correspond to the patterns that one might see visually. Finally, we show how to explicitly relate the desired frames to the averaged Tisserand frame and the geometric phase. We do the latter in case the symmetry group is abelian and cyclic coordinates can be identified; the answer is identified with a geometric construction, namely that

The angular velocity of the uniformly rotating frame that evokes the patterns in the reduced space for the case of periodic orbits equals the average of the Tisserand frame velocity (the dynamic effect) minus the average velocity associated with the holonomy (the geometric effect).

We will show that this type of result also holds for certain quasiperiodic and almost periodic motions and it is reasonable to conjecture that a similar result holds for even more complicated orbits. It would be of interest in this regard to relate the work here to that of Chossat and Golubitsky [1988], Melbourne, Dellnitz, and Golubitsky [1993], and related papers.

\section{Systems with a General Continuous Symmetry Group}

Let $G$ be a Lie group acting on a manifold $M$ on the left and let $X$ be a smooth $G$-invariant vector field (i.e., an equivariant section of the tangent bundle). The flow of $X$, obtained by solving the differential equation

$$
\dot{x}=X(x),
$$

produces a one parameter group of equivariant maps $F_{t}: M \rightarrow M$. We assume that this flow is complete for simplicity. Being equivariant, it induces a reduced flow on the orbit space: $\phi_{t}: M / G \rightarrow$ $M / G$. Thus, $\pi \circ F_{t}=\phi_{t} \circ \pi$, where $\pi: M \rightarrow M / G$ is the projection.

In this general context, if $S \subset M / G$ is an equilibrium or, respectively a periodic orbit in $M / G$, then any dynamical orbit in the set $\pi^{-1}(S) \subset M$ is called a relative equilibrium in $M$, respectively a relative periodic orbit. We will use similar terms when the orbit in the quotient space is quasiperiodic or almost periodic.

This paper is concerned with the visualization of these types of orbits for general mechanical systems with symmetry. The case of a relative equilibrium is particularly simple, as in the example of the double spherical pendulum. If $z(t) \in M$ is a relative equilibrium, then we can write

$$
z(t)=\exp (t \xi) z_{0}
$$

for some $\xi \in \mathfrak{g}$ and some $z_{0} \in M$, where $\mathfrak{g}$ is the Lie algebra of the group $G$. In a frame moving with the constant angular velocity $\xi$, the motion will appear stationary. The phrase "motion relative to a uniformly rotating frame with angular velocity $\xi$ "simply means that we replace a given orbit $z(t)$ by the new curve $\exp (-t \xi) z(t)$. "Uniformly" refers to the fact that $\xi$ is time independent. We are mainly concerned with orbits that are periodic or quasiperiodic in the reduced space. We claim that under suitable hypotheses, the motion in the original space relative to a suitably chosen uniformly rotating frame is also periodic or quasiperiodic.

In many interesting mechanical systems, there is an additional symmetry (often discrete) for the given system and this symmetry passes to the reduced space. We will show explicitly how to do this for the double spherical pendulum problem. This discrete symmetry often means that the periodic or quasiperiodic orbit on the reduced space has a discrete "space time" symmetry, as in, for example, Montaldi, Roberts and Stewart $[1988,1990]$. When one does the visualization of orbits in the original 
space by passing to a uniformly rotating frame, we want to not only capture the periodicity (not "corrupted" by the given symmetry group $G$ ) but the patterns present in the reduced space because of the discrete symmetry. This is why we call this type of construction pattern evocation.

The double spherical pendulum fits into this framework as follows. In the context of Lagrangian mechanics, we choose $M$ to be $T Q$ where $Q=S^{2} \times S^{2}$ and choose the continuous symmetry group to be $S^{1}$. The discrete symmetry group for this system was described in the introduction and will be made explicit later in $\S 5$. The overall symmetry of the system will turn out to be the orthogonal group $O(2)$. In the reduction process, we only use the connected component of the identity, namely $S^{1}$. The system has four degrees of freedom, but the reduced system (whose phase space is the $S^{1}$ orbit space of a level set of the angular momentum) has three degrees of freedom. Periodic motion in the reduced space corresponds to motion on a two torus in the original space, while motion on a two torus in the reduced space corresponds to motion on a three torus in the original space. Here the visualization process is very concrete as we have mentioned in the introduction: one looks at the motion of the pendulum bobs through the lens of an overhead rotating camera. If one has motion that is periodic or quasiperiodic (with a certain number of independent frequencies) in the reduced space then the motion in the camera will appear this way as well if its angular velocity is suitably chosen, but the dynamical motion may appear more complicated when viewed from other rotating frames.

\subsection{Existence of Uniformly Rotating Frames for Relatively Periodic Or- bits}

Let $c(t)$ be an integral curve of $X$ and let it project to a curve $\gamma(t)$ under $\pi$; that is, $\gamma(t)=\pi(c(t))$.

Theorem 2.1 Assume that the exponential map exp $: \mathfrak{g} \rightarrow G$ is surjective. (For example, this holds if $G$ is compact, connected and abelian, that is, $G$ is a torus). Assume that $c(t)$ is a relative periodic orbit; that is, the integral curve $\gamma(t)$ is closed; denote its period by $T$. Then there is a Lie algebra element $\xi \in \mathfrak{g}$ such that $\exp (-t \xi) c(t)$ is also periodic with period $T$.

Proof Since $c(t)$ covers $\gamma(t)$, and the latter is periodic with period $T$, there is a Lie group element $g \in G$ such that $c(T)=g \cdot c(0)$. Since the exponential map is onto, we can write $g=\exp (T \xi)$ for some fixed Lie algebra element $\xi \in \mathfrak{g}$. We claim that this $\xi$ satisfies the conditions required. To establish this, we need to show that the curve $\exp (-t \xi) c(t)$ is $T$-periodic. That is, we need to show that

$$
\exp (-(T+t) \xi) \cdot c(T+t)=\exp (-t \xi) \cdot c(t)
$$

This condition is equivalent to

$$
c(T+t)=\exp (T \xi) \cdot c(t)
$$

However, by $G$-invariance, $\exp (T \xi) \cdot c(t)$ is an integral curve of $X$. Thus, the two curves $c(T+t)$ and $\exp (T \xi) \cdot c(t)$ are both integral curves of $X$ and they agree at the point $t=0$, so they are equal by uniqueness of integral curves.

Let us now apply this result to the context of mechanical systems with symmetry. Let $P$ be a symplectic manifold on which a Lie group $G$ (with Lie algebra $\mathfrak{g}$ ) acts by symplectic transformations and that has an equivariant momentum map $\mathbf{J}: P \rightarrow \mathfrak{g}^{*}$. Here equivariance is taken with respect to the given action of $G$ on $P$ and the coadjoint action of $G$ on $\mathfrak{g}^{*}$. Fix a value $\mu$ of $\mathbf{J}$ and let $G_{\mu}$ be the isotropy group of $\mu \in \mathfrak{g}^{*}$ and let $\mathfrak{g}_{\mu}$ be its Lie algebra. The reduced space is the orbit space $P_{\mu}=\mathbf{J}^{-1}(\mu) / G_{\mu}$. Here we will assume that $\mu$ is a regular value of $J$ and that the quotient space does not have singularities. In this case, it is well known (Marsden and Weinstein [1974]) that the reduced space is a symplectic manifold in a natural way. We need not assume that $\mu$ is a regular element of $\mathfrak{g}^{*}$; however, we remark that the regular elements form an open dense set and the 
isotropy group $G_{\mu}$ of such elements is abelian. This is the Duflo-Vergne theorem; see Marsden and Ratiu [1994] for a proof and a discussion. If $G$ is compact and connected, so is $G_{\mu}$ (Guillemin and Sternberg [1984]) and so in this case, $G_{\mu}$ is generically a torus. (In fact, it is a maximal torus.)

Recall that if a given Hamiltonian $H$ is $G$-invariant, it induces a Hamiltonian, which we will call $H_{\mu}$ on the reduced space $P_{\mu}$ and that the dynamics of $H$ projects naturally to the dynamics of $H_{\mu}$.

For the case of the double spherical pendulum, the assumption of regularity means that we do not study the dynamics in a neighborhood of the straight down state. This state is singular (in several senses) and is discussed in Marsden and Scheurle [1993a]. While this state is of course very interesting, we do not discuss it in this paper.

Now consider an integral curve $c_{\mu}(t)$ of the reduced Hamiltonian vector field $X_{H_{\mu}}$ on $P_{\mu}$. With a given initial condition $z_{0} \in \mathbf{J}^{-1}(\mu)$, denote by $c(t)=F_{t}\left(z_{0}\right)$ the corresponding integral curve of $X_{H}$ such that $\pi_{\mu}(c(t))=c_{\mu}(t)$, where $\pi_{\mu}: \mathbf{J}^{-1}(\mu) \rightarrow P_{\mu}$ is the projection.

Specializing the preceding general result to the case of $M=\mathbf{J}^{-1}(\mu)$ and with the induced action of the symmetry group $G_{\mu}$, we get the following.

Theorem 2.2 Assume that the Lie group $G_{\mu}$ is such that its exponential map is surjective (which is true automatically for generic $\mu$ in the compact connected case). Assume that $c(t)$ is a relative periodic orbit; that is, the integral curve $c_{\mu}(t)$ is closed; denote its period by $T$. Then there is a Lie algebra element $\xi \in \mathfrak{g}_{\mu}$ such that $\exp (-t \xi) c(t)$ is also periodic with period $T$.

Later we will study systems with cyclic variables where there is an explicit formula for the angular velocity that produce the results of Theorem 2.2 in terms of the reduced orbit. Using this context we will be able to answer a similar question for quasiperiodic orbits.

\subsection{Reconstruction}

In what follows we shall obtain further information on the moving frame and how to choose its velocity and shall relate the result above to geometric and dynamic phases. To do this, we use a reconstruction construction for mechanical systems with symmetry and its relation to the theory of geometric phases; see Marsden [1992] and Marsden, Montgomery, and Ratiu [1990].

To achieve these goals, we relate the two integral curves $c(t)$ and $c_{\mu}(t)$ more specifically. To do so, choose a smooth curve $d(t)$ in $\mathbf{J}^{-1}(\mu)$ such that $d(0)=z_{0}$, where $z_{0}$ is the initial condition of the orbit in the original space and where $\pi_{\mu}(d(t))=c_{\mu}(t)$. Write $c(t)=\Phi_{g(t)}(d(t))$ for some curve $g(t)$ in $G_{\mu}$ to be determined, where the group action is denoted $g \cdot z=\Phi_{g}(z)$. First note that

$$
\begin{aligned}
X_{H}(c(t)) & =c^{\prime}(t) \\
& =T_{d(t)} \Phi_{g(t)}\left(d^{\prime}(t)\right)+T_{d(t)} \Phi_{g(t)} \cdot\left(T_{g(t)} L_{g(t)^{-1}}\left(g^{\prime}(t)\right)\right)_{P}(d(t)) .
\end{aligned}
$$

Since $\Phi_{g}^{*} X_{H}=X_{\Phi_{g}^{*} H}=X_{H},(2.2 .1)$ gives

$$
\begin{aligned}
d^{\prime}(t)+\left(T_{g(t)} L_{g(t)^{-1}}\left(g^{\prime}\right)(t)\right)_{P}(d(t)) & =T \Phi_{g(t)^{-1}} X_{H}\left(\Phi_{g(t)}(d(t))\right) \\
& =\left(\Phi_{g(t)}^{*} X_{H}\right)(d(t)) \\
& =X_{H}(d(t))
\end{aligned}
$$

Regarding $g(t)$ as the unknown in this equation, we solve for $g(t)$ in two steps:

Step 1 Find $\xi(t) \in \mathfrak{g}_{\mu}$ such that

$$
\xi(t)_{P}(d(t))=X_{H}(d(t))-d^{\prime}(t)
$$

Step 2 With $\xi(t)$ determined, solve the following non-autonomous ordinary differential equation on $G_{\mu}$ :

$$
g^{\prime}(t)=T_{e} L_{g(t)}(\xi(t)), \quad \text { with } \quad g(0)=e .
$$


Step 1 is typically of an algebraic nature; in coordinates, for matrix Lie groups, (2.2.3) is a matrix equation. We show below how $\xi(t)$ can be explicitly computed if a connection is given on $\mathbf{J}^{-1}(\mu) \rightarrow P_{\mu}$. With $g(t)$ determined, the desired integral curve $c(t)$ is given by $c(t)=\Phi_{g(t)}(d(t))$.

Step 2 can be carried out explicitly in the generic case that $G_{\mu}$ is abelian. Here (the connected component of the identity of) $G_{\mu}$ is a cylinder $\mathbb{R}^{p} \times \mathbb{T}^{k-p}$ and the exponential map

$$
\exp \left(\xi_{1}, \ldots, \xi_{k}\right)=\left(\xi_{1}, \ldots, \xi_{p}, \xi_{p+1}(\bmod 2 \pi), \ldots, \xi_{k}(\bmod 2 \pi)\right)
$$

is onto, so we can write $g(t)=\exp \eta(t)$, where $\eta(0)=0$. Therefore $\xi(t)=T_{g(t)} L_{g(t)^{-1}}\left(g^{\prime}(t)\right)=\eta^{\prime}(t)$ since $\eta^{\prime}$ and $\eta$ commute, i.e., $\eta(t)=\int_{0}^{t} \xi(s) d s$. Thus the solution of (2.2.4) is

$$
g(t)=\exp \left(\int_{0}^{t} \xi(s) d s\right)
$$

This reconstruction method depends on the choice of $d(t)$. With the help of additional structure, $d(t)$ can be chosen in a geometric way. What we use is a connection so that we can horizontally lift curves on the base to curves in the total space. Suppose then that the principal $G_{\mu}$ bundle $\pi_{\mu}: \mathbf{J}^{-1}(\mu) \rightarrow P_{\mu}$ is endowed with a connection $A$. That is, $A: T \mathbf{J}^{-1}(\mu) \rightarrow \mathfrak{g}_{\mu}$ is a $\mathfrak{g}_{\mu}$-valued one-form on $\mathbf{J}^{-1}(\mu) \subset P$ such that $A_{p} \cdot \xi_{P}(p)=\xi$ for all $\xi \in \mathfrak{g}_{\mu}$ and that $L_{g}^{*} A=A d_{g} \circ A$ for all $g \in G_{\mu}$.

We choose $d(t)$ to be the horizontal lift of $c_{\mu}$ passing through the point $z_{0}$. In other words, $d(t)$ satisfies $A(d(t)) \cdot d^{\prime}(t)=0, \pi_{\mu} \circ d=c_{\mu}$, and $d(0)=z_{0}$. We summarize the calculations for the reconstruction procedure as follows.

Theorem 2.3 Suppose $\pi_{\mu}: \mathbf{J}^{-1}(\mu) \rightarrow P_{\mu}$ is a principal $G_{\mu}$-bundle with a connection A. Let $c_{\mu}$ be an integral curve of the reduced dynamical system on $P_{\mu}$. Then the corresponding curve $c$ through a point $z_{0} \in \pi_{\mu}^{-1}\left(c_{\mu}(0)\right)$ of the system on $P$ is determined as follows:

i Horizontally lift $c_{\mu}$ to form the curve $d$ in $\mathbf{J}^{-1}(\mu)$ through $z_{0}$.

ii Let $\xi(t)=A(d(t)) \cdot X_{H}(d(t))$, so that $\xi(t)$ is a curve in $\mathfrak{g}_{\mu}$.

iii Solve the equation $\dot{g}(t)=g(t) \cdot \xi(t)$ with $g(0)=e$.

Then $c(t)=g(t) \cdot d(t)$ is the integral curve of the system on $P$ with initial condition $z_{0}$.

Suppose now that $c_{\mu}$ is a closed curve with period $T$; thus, both $c$ and $d$ intersect the same fiber of the bundle $\pi_{\mu}: \mathbf{J}^{-1}(\mu) \rightarrow P_{\mu}$. Write

$$
d(T)=\hat{g} \cdot d(0) \quad \text { and } \quad c(T)=h \cdot c(0)
$$

for $\hat{g}, h \in G_{\mu}$. Note that

$$
h=g(T) \hat{g} .
$$

The Lie group element $\hat{g}$ (or the Lie algebra element $\log \hat{g}$ ) is called the geometric phase. It is the holonomy of the path $c_{\mu}$ with respect to the connection $A$ and has the important property of being parameterization independent. The Lie group element $g(T)$ (or $\log g(T)$ ) is called the dynamic phase.

We recall that for compact or semi-simple $G$, the group $G_{\mu}$ is generically abelian and so the computation of $g(T)$ and $\hat{g}$ are then relatively easy, as was indicated above.

The connection $A$ can be (but does not have to be) taken to be the connection induced by the mechanical connection and a choice of Killing form on the Lie algebra. We will describe this connection explicitly in the Abelian case below. 


\subsection{The Mechanical Connection}

The basic ingredients needed to define the mechanical connection are $Q$, a configuration manifold, $\langle\langle$,$\rangle , a metric on Q$ (normally the kinetic energy), and $G$ a given group acting on $Q$ by isometries. Let $\xi \in \mathfrak{g}$ and let $\xi_{Q}$, a vector field on $Q$, be the corresponding infinitesimal generator. Recall that in this case, the momentum map $\mathbf{J}: T Q \rightarrow \mathfrak{g}^{*}$ is given by

$$
\langle\mathbf{J}(q, v), \xi\rangle=\left\langle\left\langle v, \xi_{Q}(q)\right\rangle\right\rangle
$$

that is, $J_{a}=g_{i j} v^{i} K^{j}{ }_{a}(q)$, where $\left(\xi_{Q}\right)^{i}=K^{i}{ }_{a}(q) \xi^{a}$ define the "action coefficients" $K^{i}$. The locked inertia tensor is given by $\mathbb{I}(q): \mathfrak{g} \rightarrow \mathfrak{g}^{*}$,

$$
\langle\mathbb{I}(q) \eta, \zeta\rangle=\left\langle\left\langle\eta_{Q}(q), \zeta_{Q}(q)\right\rangle\right\rangle
$$

or, in coordinates, $I_{a b}=g_{i j} K^{i}{ }_{a} K^{j}{ }_{b}$ We define $\mathcal{A}: T Q \rightarrow \mathfrak{g}$, a Lie algebra valued 1 -form, by

$$
\mathcal{A}(q, v)=\mathbb{I}(q)^{-1} J(q, v)
$$

or, in coordinates,

$$
\mathcal{A}^{a}=I^{a b} g_{i j} K_{b}^{i} v^{j}
$$

An alternative definition is to specify $\mathcal{A}_{\mu}$, the $\mu$-component of $\mathcal{A}$ in terms of the kinetic energy function $K$ by:

$$
K\left(\mathcal{A}_{\mu}(q)\right)=\inf \text { of } K \text { on } T_{q} Q \text { subject to the constraint } J=\mu .
$$

Intuitively, the mechanical connection gives the angular velocity of the locked system, as in coupled rigid bodies when one locks the joints of the system. One checks that $\mathcal{A}$ is a connection on the $G$-bundle $Q \rightarrow Q / G$, in the sense of a principal bundle connection.

\section{Systems with Cyclic Coordinates}

We now relate the above general theory to calculations that are specific to the case of systems with abelian symmetry groups. We make use of cyclic coordinates and classical notation to aid the exposition; as we shall see, the result is a recognizable answer and agrees with the general construction when combined with the reconstruction method described earlier.

\subsection{Notation}

We consider a simple mechanical system with Lagrangian $L$ of the form kinetic minus potential, where the configuration manifold $Q$ is a Riemannian manifold. Furthermore, assume that the symmetry group $G$ is abelian and in fact is a torus, and acts freely so that the quotient spaces are smooth. Following Marsden and Scheurle [1993b], we recall how to reduce the Euler-Lagrange equations for $L$ on $T Q$ to equations on the quotient space $T Q / G$. We shall identify the action of the symmetry group $G$ using a set of cyclic coordinates. In fact, assume that $G$ acts on $Q$ by

$$
x^{\alpha} \mapsto x^{\alpha}(\alpha=1, \ldots, m) \quad \text { and } \quad \theta^{a} \mapsto \theta^{a}+\varphi^{a}(a=1, \ldots k)
$$

where $\varphi^{a} \in[0,2 \pi)$, and where $x^{1}, \ldots, x^{m}, \theta^{1}, \ldots, \theta^{k}$ are suitably chosen (local) coordinates on $Q$. Then $G$-invariance implies that the Lagrangian $L=L(x, \dot{x}, \dot{\theta})$ does not explicitly depend on the variables $\theta^{a}$, i.e., these variables are cyclic. In these coordinates we have

$$
L(x, \dot{x}, \dot{\theta})=\frac{1}{2} g_{\alpha \beta}(x) \dot{x}^{\alpha} \dot{x}^{\beta}+g_{a \alpha}(x) \dot{x}^{\alpha} \dot{\theta}^{a}+\frac{1}{2} g_{a b}(x) \dot{\theta}^{a} \dot{\theta}^{b}-V(x) .
$$


Moreover, the infinitesimal generator $\xi_{Q}$ on $Q$ of an element $\xi=\left(\xi^{1}, \ldots, \xi^{k}\right)$ is given by

$$
\xi_{Q}=\left(0, \ldots, 0, \xi^{1}, \ldots, \xi^{k}\right)
$$

where there are $m$ zeros. Therefore we have the following action coefficients:

$$
K^{\beta}{ }_{a}=0, \quad K^{b}{ }_{a}=\delta^{b}{ }_{a} .
$$

The components of the momentum map $\mathbf{J}: T Q \rightarrow \mathfrak{g}^{*}$ for $G$ are given by

$$
J_{a}=\frac{\partial L}{\partial \dot{\theta}^{a}}=g_{a \alpha} \dot{x}^{\alpha}+g_{a b} \dot{\theta}^{b},
$$

i.e., they are the classical momenta conjugate to the variables $\dot{\theta}^{a}$. Note that the momentum map is $G$-equivariant and in fact invariant here, and that its components are conserved quantities for the equations of motion. Thus, the level surfaces of $\mathbf{J}$ in $T Q$ are invariant. In the present coordinates this also follows immediately from the standard Euler-Lagrange equations for $L$

$$
\begin{aligned}
\frac{d}{d t}\left(\frac{\partial L}{\partial \dot{x}^{\gamma}}\right)-\frac{\partial L}{\partial x^{\gamma}} & =0 \quad(\gamma=1, \ldots, m) \\
\frac{d}{d t}\left(\frac{\partial L}{\partial \dot{\theta}^{c}}\right) & =0 \quad(c=1, \ldots, k) .
\end{aligned}
$$

It is readily checked that the locked inertia tensor $\mathbb{I}_{a b}$ is given by $g_{a b}$. We denote the inverse of this tensor by $\mathbb{I}^{a b}=g^{a b}$.

Note that (3.1.2) are local equations, since the coordinates $x, \dot{x}, \dot{\theta}$ correspond to a (local) trivialization of the bundle $T Q / G$. Since these equations are independent of $\theta$, they locally drop to the quotient. In order to give the dropped equations a global (intrinsic) meaning, we now introduce the velocity shift. To this end we replace the variables $\dot{\theta}^{a}$ by $\xi^{a}$ given by means of the mechanical connection $\mathcal{A}$ as follows:

$$
\begin{aligned}
\xi^{a} & =\mathbb{I}^{a b} g_{i j} K^{i} \dot{q}^{j} \\
& =\mathbb{I}^{a b} g_{b j} \dot{q}^{j} \\
& =\mathbb{I}^{a b} g_{b \alpha} \dot{x}^{\alpha}+\mathbb{I}^{a b} g_{b c} \dot{\theta}^{c} \\
& =\dot{\theta}^{a}+\mathcal{A}_{\alpha}^{a} \dot{x}^{\alpha},
\end{aligned}
$$

where

$$
\mathcal{A}_{\alpha}^{a}=\mathbb{I}^{a b} g_{b \alpha}
$$

are the components of the connection. Thus,

$$
\dot{\theta}^{a}=\dot{\tilde{\theta}}(x, \dot{x}, \xi)=\xi^{a}-\mathcal{A}_{\alpha}^{a} \dot{x}^{\alpha},
$$

which defines $\dot{\theta}$ in terms of $(x, \dot{x}, \xi)$. In particular, as a Lie algebra valued 1-form, $\mathcal{A}$ has components

$$
\mathcal{A}^{a}=d \theta^{a}+\mathcal{A}_{\alpha}^{a} d x^{\alpha} .
$$

Thus, we replace $\dot{\theta}$ by the locked angular velocity $\xi \in \mathfrak{g}$, the generator $\xi_{Q}$ of which gives the velocity component tangential to the group orbit at a given configuration of the system. In terms of the transformed Lagrangian

$$
l(x, \dot{x}, \xi)=L(x, \dot{x}, \dot{\tilde{\theta}}(x, \dot{x}, \xi))
$$


the equations of motion reads as follows

$$
\begin{aligned}
\frac{d}{d t} \frac{\partial l}{\partial \dot{x}^{\gamma}}-\frac{\partial l}{\partial x^{\gamma}} & =\frac{\partial l}{\partial \xi^{a}} \mathcal{B}_{\gamma \alpha}^{a} \dot{x}^{\alpha} \quad(\gamma=1, \ldots, m) \\
\frac{d}{d t} \frac{\partial l}{\partial \xi^{c}} & =0 \quad(c=1, \ldots, k)
\end{aligned}
$$

as a straightforward computation using the chain rule shows. These equations are a special case of the general reduced Euler-Lagrange equations of Marsden and Scheurle [1993b]. Here

$$
\mathcal{B}_{\gamma \alpha}^{a}=\mathcal{A}_{\alpha, \gamma}^{a}-\mathcal{A}_{\gamma, \alpha}^{a}
$$

can be viewed as the components of the curvature $\mathcal{B}$ of the connection $\mathcal{A}$ which in the abelian case, is given by the exterior derivative of $\mathcal{A}$ :

$$
\mathcal{B}^{a}=[\mathbf{d} \mathcal{A}]^{a}=\sum_{\gamma<\alpha} \mathcal{B}_{\gamma \alpha}^{a} d x^{\gamma} \wedge d x^{\alpha} .
$$

Thus, the first set of equations in (3.1.3) are forced Euler-Lagrange equations with Lagrangian $l$ as a function of the base variables $(x, \dot{x})$. The second set of equations are Euler-Poincaré equations in $\xi$, which are degenerate because we are dealing with an abelian group $G$ here. Since these equations as well as $l$ do not depend on the $\theta$-variables, they drop to the quotient $(T Q) / G$ just as they are stated.

In Marsden and Scheurle [1993a], we carried out a further reduction to $J^{-1}(\mu) / G_{\mu}$ in the case of a simple mechanical system and assuming that the quotient is a smooth manifold again, i.e., $\mu$ is a regular value of $J$ and $G_{\mu}$ acts freely on the level set $J=\mu$. Recall that $G_{\mu}$ is the isotropy subgroup of $\mu$. For abelian groups, locally, i.e., in coordinates, this reduction amounts to the classical Routhian procedure, which leads to Euler-Lagrange equations for the classical Routhian $R_{\text {class }}^{\mu}$. Note that in the abelian case, $G_{\mu}=G$ and $J^{-1}(\mu) / G_{\mu} \approx T(Q / G)$. Marsden and Scheurle [1993a] modified this procedure to give it an intrinsic (global) meaning and to include the case of nonabelian groups $G$. In terms of the setting of this section, the Routhian $R^{\mu}$ is defined by a (partial) Legendre transformation of $l$ with respect to $\xi$ :

$$
R^{\mu}(x, \dot{x})=[l(x, \dot{x}, \xi)-\langle\mu, \xi\rangle]_{\mid \xi=\tilde{\xi}(x, \dot{x}, \mu)}
$$

where $\tilde{\xi}(x, \dot{x}, \mu)$ is the unique solution for $\xi$ of the equation

$$
\frac{\partial l}{\partial \xi}(x, \dot{x}, \xi)=\mu
$$

for a particular value of $\mu \in \mathfrak{g}^{*}$. So, now we replace the variable $\xi$ by $\mu$ in the equations (3.1.3). Then the second set of equations in (3.1.3) just become

$$
\frac{d}{d t} \mu_{c}=0 \quad(c=1, \ldots, k)
$$

which expresses the fact, that $\mu$ is conserved for the motion. Thus, fixing a particular value for $\mu$, the restriction of (3.1.2) to the level set $J=\mu$ is given by the first set of equations in (3.1.3). Rewriting those in terms of the Routhian $R^{\mu}$ rather than $l$ leads to

$$
\frac{d}{d t} \frac{\partial R^{\mu}}{\partial \dot{x}^{\gamma}}-\frac{\partial R^{\mu}}{\partial x^{\gamma}}=\mu_{a} \mathcal{B}_{\gamma \alpha}^{a} \dot{x}^{\alpha}=\left[\mathbf{i}_{\dot{x}} \mathcal{B}_{\mu}\right]_{\gamma} \quad(\gamma=1, \ldots, m),
$$

i.e., on $J=\mu$ we have Euler-Lagrange equations for the Routhian $R^{\mu}$ with forcing given by the magnetic 2 -form $\mathcal{B}_{\mu}$ which in the present coordinates is defined as follows:

$$
\mathcal{B}_{\mu}=\sum_{\gamma<\alpha} \mu_{a} \mathcal{B}_{\gamma \alpha}^{a} d x^{\gamma} \wedge d x^{\alpha} .
$$


Note that $\mathcal{B}_{\mu}=d \mathcal{A}_{\mu}$ is the exterior derivative of the 1 -form $\mathfrak{A}_{\mu}: Q \rightarrow T^{*} Q$ given by

$$
\mathcal{A}_{\mu}=\mu_{a} d \theta^{a}+\mu_{a} \mathcal{A}_{\alpha}^{a} d x^{\alpha} .
$$

Note that in contrast to $\mathcal{A}_{\mu}, \mathcal{B}_{\mu}$ does not depend on the $\theta$-variables just as $R^{\mu}$ does not. Therefore all terms in (3.1.4) drop further to the quotient $J^{-1}(\mu) / G$ and the structure of these equations is preserved there.

Moreover, locally these equations are even standard Euler-Lagrange equations on the quotient. Namely, as a closed 2-form $\mathcal{B}_{\mu}$ is locally exact. Therefore, locally the right-hand side of (3.1.4) can be included into the left-hand side by replacing $R^{\mu}$, by the classical Routhian

$$
\begin{aligned}
R_{\text {class }}^{\mu} & =\left[L(x, \dot{x}, \dot{\theta})-\mu_{a} \dot{\theta}^{a}\right]_{\dot{\theta}^{a}=\dot{\theta^{\alpha}}(x, \dot{x}, \mu)} \\
& =R^{\mu}+\mu_{a} g_{\alpha a} \dot{x}^{\alpha} \mathbb{I}^{c a}
\end{aligned}
$$

where

$$
\dot{\theta}^{a}=\dot{\tilde{\theta^{a}}}(x, \dot{x}, \mu)=\left[\mu_{c}-g_{\alpha c} \dot{x}^{\alpha}\right] \mathbb{I}^{c a}
$$

is the unique solution of the equation $J=\mu$ with respect to $\dot{\theta}$. It is to be noted that globally, $\mathcal{B}_{\mu}$ is not exact on the quotient $J^{-1}(\mu) / G$ in general. In the present context this can be seen from the fact that the 1-form $\mathcal{A}_{\mu}$ explicitly depends on the $\theta$-variables.

We refer to Marsden and Scheurle [1993a] for a Lagrange-d'Alembert variational principle behind the equations (3.1.4). We also point out that there are variational principles underlying the general reduced Euler-Lagrange equations as well, as has been shown by Marsden and Scheurle [1993b].

\subsection{The Frame Angular Velocity Formula}

In the context of the preceding section, we give the formula for the angular velocity $\xi^{a}$ of the frame that does the job of pattern evocation. We state this in the following way:

Theorem 3.1 Using the above notation, suppose that $q(t)$ is a solution of the Euler-Lagrange equations on $Q$ and that the reduced trajectory $x(t)$ is periodic with period $T$. Then relative to the frame with angular velocity given by

$$
\xi^{a}=\frac{1}{T} \int_{0}^{T} \mathbb{I}^{c a}(x(t)) \mu_{c} d t-\frac{1}{T} \int_{0}^{T} g_{\alpha c}(x(t)) \dot{x}^{\alpha}(t) \mathbb{I}^{c a}(x(t)) d t,
$$

the solution $q(t)$ is also periodic with period $T$.

We observe that the first term is the average of the locked velocity, so represents the dynamic phase. The second term, which can also be written as a loop integral

$$
\frac{1}{T} \oint \mathcal{A}_{\alpha}^{a} d x^{\alpha}
$$

represents the effective angular velocity for the geometric phase.

To prove the theorem, we need only show that $\theta^{a}-\xi^{a} t$ is periodic with period $T$. We do this as a consequence of the following standard lemma

Lemma 3.2 Suppose that $f(s)$ is a periodic function with period $T$. Then the function

$$
F(t)=\int_{0}^{t} f(s) d s-\frac{t}{T} \int_{0}^{T} f(s) d s
$$

is periodic with period $T$. 
Proof We have

$$
\begin{aligned}
F(T+t)-F(t) & =\int_{0}^{T+t} f(s) d s-\frac{t+T}{T} \int_{0}^{T} f(s) d s-\int_{0}^{t} f(s) d s+\frac{t}{T} \int_{0}^{T} f(s) d s \\
& =\int_{t}^{T+t} f(s) d s+\left(\frac{t}{T}-\frac{t+T}{T}\right) \int_{0}^{T} f(s) d s \\
& =\int_{t}^{T+t} f(s) d s-\int_{0}^{T} f(s) d s=0
\end{aligned}
$$

This proves the lemma.

Proof of Theorem Use our prior formula for the momentum map to give

$$
\mu_{a}=g_{a \alpha} \dot{x}^{\alpha}+g_{a b} \dot{\theta}^{b} .
$$

Solving for $\dot{\theta}^{a}$ and integrating gives

$$
\theta^{b}(t)-\theta^{b}(0)=\int_{0}^{t} \mathbb{I}^{a b}(x(s)) \mu_{a} d s-\int_{0}^{t} \mathbb{I}^{a b}(x(s)) g_{a \alpha}(x(s)) \dot{x}^{\alpha}(x(s)) d s .
$$

Now we apply the lemma, for each fixed $b$, to the function

$$
f(s)=\mathbb{I}^{a b}(x(s)) \mu_{a}-\mathbb{I}^{a b}(x(s)) g_{a \alpha}(x(s)) \dot{x}^{\alpha}(x(s)),
$$

which gives the desired result.

\subsection{The Quasiperiodic Case}

Here we generalize the result of Theorem 3.1 to the case where the reduced orbit $x(t)$ is quasiperiodic with $n$ independent frequencies $\omega_{1}, \ldots, \omega_{n} \in \mathbb{R}$, i.e., there exists a function $\phi=\phi\left(z_{1}, \ldots, z_{n}\right)$ which is $2 \pi$ periodic in all its arguments, such that

$$
x(t)=\phi\left(\omega_{1} t, \ldots, \omega_{n} t\right)
$$

holds for all $t$. It turns out that the straightforward generalization of the formula for the angular velocity $\xi^{a}$ in Theorem 7.1 leads to the desired result, provided that the frequencies $\omega_{1}, \ldots, \omega_{n}$ satisfy a nonresonance condition of KAM type:

$$
\left|\sum_{k=1}^{n} j_{k} \omega_{k}\right| \geq \gamma\|j\|^{-\tau}
$$

for all nonzero $j=\left(j_{1}, \ldots j_{n}\right) \in \mathbb{Z}^{n}$. Here $\gamma$ and $\tau$ are positive constants, and $\|\cdot\|$ is any chosen norm in $\mathbb{R}^{n}$. It is well known that with respect to the Lebesgue measure, almost all $n$-tuples of frequencies $\left(\omega_{1}, \ldots, \omega_{n}\right) \in \mathbb{R}^{n}$ satisfy such a condition.

If $x(s)$ is quasiperiodic, then the functions $f(s)$ in (3.2.1) are also quasiperiodic with the same frequencies. Thus, they can be represented by their Fourier series:

$$
\sum_{j \in \mathbb{Z}^{n}} a_{j} e^{i(j \cdot \omega) t}
$$

where $\omega=\left(\omega_{1}, \ldots, \omega_{n}\right)$ is the frequency vector, $j \cdot \omega$ is the Euclidean inner product, and the Fourier coefficients are given by

$$
a_{j}=\lim _{T \rightarrow \infty} \frac{1}{T} \int_{0}^{T} f(s) e^{-i(j \cdot \omega) s} d s .
$$


If $f$ is $C^{k}$ for $k \geq \tau+2$, then there is a positive constant $\mu$ such that

$$
\left|a_{j}\right| \leq \mu\|j\|^{-(\tau+2)}
$$

for all nonzero $j \in \mathbb{Z}^{n}$. We will use this to obtain the following result.

Theorem 3.3 Suppose that $q(t)$ is a solution of the Euler Lagrange equations on $Q$ and that the reduced trajectory $x(t)$ is quasiperiodic with the frequencies $\omega_{1}, \ldots, \omega_{n}$ such that (3.3.1) holds. Assume that the metric tensor and the potential are $C^{k}$ for $k \geq \tau+2$. Then, relative to the frame with angular velocity given by the formula

$$
\xi^{a}=\lim _{T \rightarrow \infty} \frac{1}{T} \int_{0}^{T}\left[\mathbb{I}^{c a}(x(t)) \mu_{c}-g_{\alpha c}(x(t)) \dot{x}^{\alpha}(t) \mathbb{I}^{c a}(x(t))\right] d t
$$

the solution $q(t)$ is also quasiperiodic with the frequencies $\omega_{1}, \ldots, \omega_{n}$.

Proof Analogously to the periodic case, to prove this theorem, we need to show that $\theta^{a}-\xi^{a} t$ is quasiperiodic with the frequencies $\omega_{1}, \ldots, \omega_{n}$. Noting that the integrand in the preceding formula is $C^{k}$, this follows, as in the periodic case, as a consequence of a standard lemma:

Lemma 3.4 Suppose that $f(s)$ is quasiperiodic with frequencies $\omega_{1}, \ldots, \omega_{n}$ such that (3.3.1) holds. Furthermore, suppose that the Fourier coefficients of $f$ satisfy (3.3.2). Then the function

$$
F(t)=\int_{0}^{t} f(s) d s-t \cdot \lim _{T \rightarrow \infty} \frac{1}{T} \int_{0}^{T} f(s) d s
$$

is quasiperiodic with the frequencies $\omega_{1}, \ldots, \omega_{n}$.

Proof Represent $f$ by its Fourier series

$$
f(s)=\sum_{j \in \mathbb{Z}^{n}} a_{j} e^{i(j \cdot \omega) s},
$$

and note that the second term in the formula for $F(t)$ is just $t a_{0}$ where

$$
a_{0}=\lim _{T \rightarrow \infty} \frac{1}{T} \int_{0}^{T} f(s) d s
$$

is the mean value of $f$. Hence, $F(t)$ is given by

$$
F(t)=\int_{0}^{t}\left(\sum_{j \neq 0} a_{j} e^{i(j \cdot \omega) s}\right) d s .
$$

By (3.3.2), the Fourier series for $f$ is uniformly convergent with respect to $s$ and hence we can integrate termwise, yielding

$$
F(t)=\sum_{j \neq 0} \frac{a_{j}}{i(\omega \cdot j)} e^{i(\omega \cdot j) t} .
$$

But, due to the nonresonance condition (3.3.1) and (3.3.2), the series

$$
\phi(z)=\sum_{j \neq 0} \frac{a_{j}}{i(\omega \cdot j)} e^{i(j \cdot z)},
$$

converges uniformly in $z=\left(z_{1}, \ldots z_{n}\right)$ and the limit function $\phi(z)$ is $2 \pi$-periodic in all of its arguments $z_{i}$. By construction, $F(t)=\phi(\omega t)$ and therefore $F(t)$ is quasiperiodic with frequencies $\omega_{1}, \ldots, \omega_{n}$. 


\section{Remarks}

1. Note that there is a necessary as well as sufficient condition for $F$ in (3.3.4) to be quasiperiodic with the same frequencies as $f$, namely uniform boundedness of $F$ with respect to $t \in \mathbb{R}$. Here no non-resonance condition of type (3.3.1) is needed. Furthermore, this criterion carries over directly to the case of a general almost periodic function $f$. Hence, our theory makes sense even in certain cases when the reduced orbit is almost periodic but not quasiperiodic. Recall that a continuous function $f(t)$ is said to be almost periodic if the following holds: Given any positive real number $\epsilon$, there is a positive real number $T=T(\epsilon)$ such that any interval of length $T$ on the real axis contains a number $\tau$ with $|f(t+\tau)-f(t)| \leq \epsilon$ for all $t \in \mathbb{R}$. Each almost periodic function $f(t)$ has a Fourier expansion of the form

$$
f(t)=\sum_{j \in \mathbb{Z}} a_{j} e^{i \omega_{j} t}
$$

where the frequencies $\omega_{j}$ are certain real numbers and the coefficients $a_{j}$ are defined in a way analogous to the quasiperiodic case (see, for example, Bohr [1947]).

Of course, in general it is not easy to check the boundedness of $F$. In fact, there are many combinations of frequencies of $f$ such that $F$ fails to be bounded. For example, in the quasiperiodic case, the set of $n$-tuples of frequencies $\left(\omega_{1}, \ldots, \omega_{n}\right)$ in $\mathbb{R}^{n}$ for which $F$ is unbounded, is of the second category (see Scheurle [1981]).

2. Despite the preceding remark, our formula for $\xi^{a}$ can always be used to visualize quasiperiodicity or almost periodicity of the reduced orbit $x(t)$ in the following approximate sense. Given any positive real numbers $\epsilon$ and $T$, then relative to the rotating frame with $\xi^{a}$ as in (3.3.3), and for $0 \leq t \leq T$, the exact orbit $q(t)$ is $\epsilon$-close to an approximate orbit $\tilde{x}(t)$ which is almost periodic with the same frequencies as $x(t)$. From a practical point of view, this actually justifies our method completely. The approximate orbit $\tilde{x}(t)$ is determined by approximating $\theta^{a}-\xi^{a} t$ by a trigonometric polynomial, the derivative of which is the Fourier expansion of $\dot{\theta}^{a}-\xi^{a}$ truncated after sufficiently many terms. Here we assume that this Fourier expansion converges uniformly to $\dot{\theta}^{a}-\xi^{a}$.

3. We finally consider the special case where the quotient $Q / G$ is one-dimensional, which implies complete integrability of the original system. First of all assume that $x$ is a real coordinate in a single coordinate chart. Then the integral of the second term of the integrand in (3.3.3) is given by the integral of $g_{a c}(x) \mathbb{I}^{c a}(x)$ evaluated along the reduced orbit $x=x(t)$. Therefore, the contribution of that second term to $\xi^{a}$ is zero, if $x(t)$ is bounded. In other words, the term in the formula for $\xi^{a}$ related to the geometric phase is zero in this case. On the other hand, if the quotient space is a circle, so that $x$ is an angular variable, the contribution from the geometric phase term can be nonzero.

\section{Discrete Symmetries}

\subsection{Introduction}

Next we study the role of discrete symmetries. We do this by enlarging the group $G$ with which we reduce to a group we will call $\Gamma$. One can view the discrete symmetry group in one of two ways. First, one can assume that $G$ is a normal subgroup of $\Gamma$ and then define the "discrete" symmetries by $\Sigma=\Gamma / G$. Alternatively, we can suppose that the discrete symmetries are given separately as a group $\Sigma$, assume that $\Sigma$ acts on $G$, and then form the semidirect product $\Sigma(S) G$. Recall that the semidirect product group has the multiplication law

$$
\left(\sigma_{1}, g_{1}\right) \cdot\left(\sigma_{2}, g_{2}\right)=\left(\sigma_{1} \sigma_{2},\left(\sigma_{2}\right)_{G}\left(g_{1}\right) g_{2}\right) \text {. }
$$


These two approaches are essentially the same; namely, given a semidirect product $\Gamma=\Sigma(S) G$, the group $G$ may be regarded as a normal subgroup of $\Gamma$ and the quotient group is $\Sigma$. Conversely, given $\Gamma$, a normal subgroup $G$, and a group homomorphism $\tau: \Gamma / G \rightarrow \Gamma$, an action of $\Gamma / G$ on $G$ is induced as well as an isomorphism between $\Gamma$ and $\Sigma($ S) $G$.

In some examples, the discrete symmetries are manifest or are readily constructed. For example, in the double spherical pendulum, we shall discuss how to use the rotational symmetry and the symmetries of reflection in vertical planes and time reversibility to obtain the enlarged group $\Gamma$. In other cases, the discrete symmetries might arise as more subtle normal form symmetries near resonances or other interesting critical phenomena.

We assume that the group $\Gamma$ acts on our phase space $P$. We are assuming that the "continuous" group $G$ acts freely (removing singular points if necessary) and that we can thereby form the quotient space $P / G$, but we do not assume that the $\Gamma$ itself acts freely since we are interested in the discrete isotropy of points in a sense to be made precise.

Assuming we have a given homomorphism $\tau: \Sigma=\Gamma / G \rightarrow \Gamma$, there is also an action of $\Sigma$ on $P$. The action of $\Sigma$ on $P$ and its action on $G$ are assumed to be compatible in a sense made precise below. Conversely, if one is given a semidirect product and an action of $\Sigma$ on $P$ compatible with its action on $G$, then one gets an action of $\Gamma$ on $P$. Generally we also want the action to have an associated equivariant momentum map as well; we will spell out this aspect below as well.

Another important ingredient for the examples is to include some aspects of reversibility. Reversible transformations are usually antisymplectic (or antipoisson) and transform the Hamiltonian vector field to its negative. The discrete symmetry group $\Sigma$ can, in general, include both symplectic and antisymplectic transformations. The symplectic transformations define a normal subgroup $\Sigma_{s}$ of $\Sigma$ and the antisymplectic transformations are given by $\Sigma_{a}=\Sigma \backslash \Sigma_{s}$.

The discrete group $\Gamma$ induces an action on the quotient phase space $P / G$ and provides "spatiotemporal" structure to solutions in the reduced space. For example, a periodic orbit could have the symmetry of this discrete group; that is, be invariant under the discrete group up to a temporal phase shift. We want to show in what sense this symmetry can be visualized in the original phase space.

\subsection{The relation between quotient groups and semidirect products}

We now show how to relate the two approaches mentioned above. We start by assuming that we have a semidirect product $\Sigma(S) G$ where, in many cases, $\Sigma$ is the discrete group, and $G$ is the continuous group of interest. A simple example is $O(2)$ regarded as the semidirect product $\mathbb{Z}_{2}$ (S) $S O(2)$, with $\mathbb{Z}_{2}$ regarded as reflections in a fixed line in $\mathbb{R}^{2}$ and acting on $S O(2)$ by conjugation.

We now want to give the assumptions explicitly that enable one to extend the action of $G$ on our phase space $P$ to an action of $\Gamma=\Sigma$ (S) $G$ on $P$.

Let $(P, \Omega)$ be a symplectic manifold, $G$ a Lie group acting on $P$ by symplectic transformations and $\mathbf{J}: P \rightarrow \mathfrak{g}^{*}$ an $\mathrm{Ad}^{*}$-equivariant momentum map for the $G$-action. Let $\Sigma$ be a Lie group acting on $P$ and each such transformation is either symplectic or antisymplectic; we then form the subgroup $\Sigma_{s}$ and the set $\Sigma_{a}$ as above. We also assume that $\Sigma$ acts on $G$ by group homomorphisms. For $\sigma \in \Sigma$, write

$$
\sigma_{P}: P \rightarrow P \quad \text { and } \quad \sigma_{G}: G \rightarrow G
$$

for the corresponding symplectic map on $P$ and group homomorphism on $G$. Let $\sigma_{\mathfrak{g}}: \mathfrak{g} \rightarrow \mathfrak{g}$ be the induced Lie algebra homomorphism (the derivative of $\sigma_{G}$ at the identity) and $\sigma_{\mathfrak{g}^{*}}: \mathfrak{g}^{*} \rightarrow \mathfrak{g}^{*}$ be the dual of $\left(\sigma^{-1}\right)_{\mathfrak{g}}$, so, as is well known, $\sigma_{\mathfrak{g}^{*}}$ is a Poisson map with respect to the Lie-Poisson structure. See, for example, Marsden and Ratiu [1994].

Assumption 1 The actions of $G$ and of $\Sigma$ are compatible in the sense that the following equation holds:

$$
\sigma_{P} \circ g_{P}=\left[\sigma_{G}(g)\right]_{P} \circ \sigma_{P}
$$


for each $\sigma \in \Sigma$ and $g \in G$, where we have written $g_{P}$ for the action of $g \in G$ on $P$. (See Figure 4.2.1).

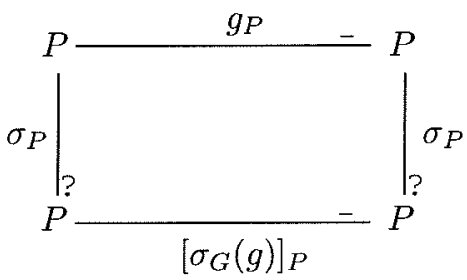

Figure 4.2.1: Assumption 1: Compatibility of the discrete and the continuous actions.

This is the key property by which an action of $\Gamma$ can be constructed. Conversely, if one starts with an action of $\Gamma$ and if a homomorphism $\tau$ is chosen, then $\Sigma$ inherits the properties in Assumption 1.

\subsection{Preservation properties of the momentum map}

Next we turn to the properties of the momentum map. If we differentiate equation (4.2.1) with respect to $g$ at the identity $g=e$, in the direction $\xi \in \mathfrak{g}$, we get

$$
T \sigma_{P} \circ X_{\langle J, \xi\rangle}=X_{\left\langle J, \sigma_{\mathfrak{g}} \cdot \xi\right\rangle} \circ \sigma_{P}
$$

where $X_{f}$ denotes the Hamiltonian vector field on $P$ generated by the function $f: P \rightarrow \mathbb{R}$. Since $\sigma_{P}$ is symplectic (respectively antisymplectic) for $\sigma \in \Sigma_{s}$ (respectively $\sigma \in \Sigma_{a}$ ), (4.3.1) is equivalent to

$$
X_{\langle J, \xi\rangle \circ \sigma_{P}^{-1}}= \pm X_{\left\langle J, \sigma_{\mathfrak{g}} \cdot \xi\right\rangle}
$$

i.e.,

$$
\mathbf{J} \circ \sigma_{P}= \pm \sigma_{\mathfrak{g}^{*}} \circ \mathbf{J}+(\text { cocycle }) \text {. }
$$

We shall assume, in addition, that the cocycle is zero. In other words, we make:

Assumption 2 The following equation holds (see Figure 4.3.1):

$$
\mathbf{J} \circ \sigma_{P}= \pm \sigma_{\mathfrak{g}^{*}} \circ \mathbf{J},
$$

where we use the plus sign for symplectic transformations and the minus for the antisymplectic case.

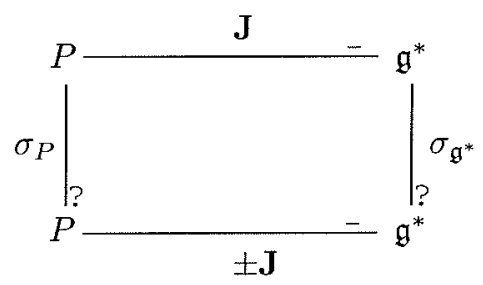

Figure 4.3.1: Assumption 2: Compatibility of the discrete action with the momentum map.

Assumption 1 shows that there is a well defined action of $\Sigma$ on $P / G$. From Assumption 2, and the additional assumption that $\Sigma$ preserves $\mathbf{J}$, there is a well defined action on the symplectic reduced space. 


\subsection{Relative Equilibria}

We begin with the simplest case, namely that of relative equilibria to fix the ideas. Thus, let $w \in P / G$ be a fixed point in the quotient space, so that the integral curve $c(t)$ that projects to $w$ is a relative equilibrium. We assume that $w$ has a discrete symmetry $\sigma_{0} \in \Sigma$, which means that

$$
\sigma_{0} w=w
$$

There is a unique Lie algebra element $\xi$ such that $c(t)=e^{\xi t} c(0)$. This Lie algebra element plays the role of the angular velocity determined by our procedures for periodic, quasiperiodic, or almost periodic orbits.

In this context, when we pass to the frame rotating with angular velocity $\xi$, we obtain the "visualized orbit" $c(0)$. However, this point in $P$ need not have the discrete symmetry $\sigma_{0}$ because the continuous symmetry $G$ is not "synchronized" with the discrete symmetry. For example, if $G=S O(2)$ acts by rotations about the $z$-axis and $\Sigma=\mathbb{Z}_{2}$ acts by reflections in a vertical plane, then a point in the original phase space needs to be rotated into the plane of reflection before it is fixed by a particular reflection $\sigma_{0}$.

Generally, it follows that $c(0)$ has at least some symmetry which is related to $\sigma_{0}$. In fact, since (4.4.1) holds, and $\sigma_{0} c(0)$ and $c(0)$ project down to $\sigma_{0} w$ and $w$ respectively, we have

$$
\sigma_{0} c(0)=g_{0} c(0)
$$

for some group element $g_{0} \in G$. Here we make use of a given homomorphism $\tau: \Sigma \rightarrow \Gamma$ to regard $\sigma_{0}$ as an element of $\Gamma$. Hence $c(0)$ is fixed under the group element $g_{0}^{-1} \sigma_{0} \in \Gamma$, i.e.,

$$
g_{0}^{-1} \sigma_{0} c(0)=c(0) \text {. }
$$

On the other hand, one can now try to synchronize the continuous symmetry group $G$ with the particular discrete symmetry $\sigma_{0}$ by shifting the "visualized" orbit $c(0)$ to the point $z=g_{1} c(0)$, where the group element $g_{1} \in G$ is determined by the requirement that $\sigma_{0} z=z$, i.e., $\sigma_{0} g_{1} c(0)=g_{1} c(0)$. By (4.4.2), this leads to the following commutator equation

$$
\sigma_{0} g_{1}^{-1} \sigma_{0}^{-1} g_{1}=g_{0}
$$

to be solved for $g_{1}$. Thus, if this equation has a solution for $g_{1} \in G$, then the "synchronized visualized" orbit $z$ has the same symmetry $\sigma_{0}$ as $w$, the reduced orbit corresponding to $c(t)$.

An equivalent way to interpret $\sigma_{0} g_{1} c(0)=g_{1} c(0)$ is that $c(0)$ has the symmetry $g_{1}^{-1} \sigma_{0} g_{1}$ which is conjugate to $\sigma_{0}$ and hence has the same group theoretical properties as $\sigma_{0}$. For instance, in the case of the above example, with $G=S O(2)$ and $\Sigma=\mathbb{Z}_{2}, g_{1}^{-1} \sigma_{0} g_{1}$ is a reflection in the vertical plane rotated by $g_{1}$.

\section{Remarks}

1. If $\sigma_{0}(g)=g^{-1}$, then one can choose $g_{1}$ to be a square root of $g_{0}$.

2. To see that (4.4.3) is solvable for $O(2)$, let $\sigma_{0}$ be the reflection in the $x$-axis in $\mathbb{R}^{2}$ and $g_{0}$ be a rotation through an angle $\theta_{0}$. Then the preceding remark applies and so $g_{1}$ may be chosen to be the rotation through an angle $\theta_{0} / 2$ or $\theta_{0} / 2+\pi$.

\subsection{General Time Dependent Orbits}

Now let $w(t) \in P / G$ be a time dependent orbit, for example, a periodic, quasiperiodic, or almost periodic orbit. Assume that it has a discrete symmetry $\left(\sigma_{0}, l_{0}\right) \in \Sigma \times \mathbb{R}$, where $\mathbb{R}$ acts on orbits by time shifts, i.e.,

$$
\sigma_{0} w( \pm t)=w\left(t+l_{0}\right)
$$


for all $t \in \mathbb{R}$. Here the plus sign refers to the case where $\sigma_{0} \in \Sigma_{s}$ is symplectic, whereas the minus sign refers to the case where $\sigma_{0} \in \Sigma_{a}$ is antisymplectic. Let $c(t)$ be an orbit covering $w(t)$ and let

$$
\tilde{c}(t)=\exp (-\xi t) c(t)
$$

be the "visualized" orbit, as constructed earlier, so that $\tilde{c}(t)$ has the same dynamical properties as $w(t)$, such as periodicity, quasiperiodicity, or almost periodicity.

We claim that

$$
\sigma_{0} \tilde{c}( \pm t)=g_{0} \tilde{c}\left(t+l_{0}\right)
$$

holds for some $g_{0} \in G$ and for all $t \in \mathbb{R}$ provided that the momentum map $\mathbf{J}$ is $\sigma_{0}$-invariant. We note right away, that $\sigma_{0}$ can preserve $\mathbf{J}$ even if it is antisymplectic, as we shall see in the double spherical pendulum example. To prove (4.5.3), first note that both sides project to the same element in the quotient at $t=0$. This defines $g_{0}$. Second, it is sufficient to show that both sides are integral curves of the common vector field $X_{H-\langle J, \xi\rangle}$. To show the latter, denote the action of $\exp (-\xi t)=e^{-\xi t}$ on $P$ by $\phi_{\exp (-\xi t)}$ and recall that this action is symplectic. Hence, since $c(t)$ is an integral curve of $X_{H}$, we have

$$
\begin{aligned}
\frac{d}{d t} \tilde{c}(t) & =T \phi_{\exp (-\xi t)} \cdot X_{H}(c(t))-X_{\langle\mathrm{J}, \xi\rangle}(\exp (-\xi t) c(t)) \\
& =X_{H-\langle\mathrm{J}, \xi\rangle}(\tilde{c}(t))
\end{aligned}
$$

i.e., $c(t)$ is an integral curve of $X_{H-\langle\mathrm{J}, \xi\rangle}$. Now the claim follows since this vector field is autonomous, equivariant with respect to $G$ and $\Sigma_{s}$, and reversible with respect to $\Sigma_{a}$.

Starting from (4.5.1) we can proceed as in the case of relative equilibria. Namely, (4.5.1) says that the "visualized orbit" $\tilde{c}(t)$ has the symmetry $\left(g_{0}^{-1} \sigma_{0}, l_{0}\right) \in \Gamma \times \mathbb{R}$ which is related to the symmetry $\left(\sigma_{0}, l_{0}\right)$ of the reduced orbit $w(t)$ as in the case of relative equilibria. However, assuming that the commutator equation (4.4.3) has a solution $g_{1} \in G$, we can again synchronize by shifting $\tilde{c}(t)$ to $g_{1} \tilde{c}(t)$, which then has the same symmetry $\left(\sigma_{0}, l_{0}\right)$ as the reduced orbit.

\section{Remark}

One has to be careful between the symmetries one can see in $P$ and those in $P / G$. In particular, one can have "more" symmetries for the "visualized orbits" in $P$ than in $P / G$. Specifically, it is possible that there can be many values of $\xi$ (beside the one constructed to visualize the periodicity or quasiperiodicity) and associated group elements that project to the same symmetry in the reduced space. One sees this mulitiplicity in simulations of the double spherical pendulum, for example.

\section{The Double Spherical Pendulum}

In this section we discuss the theory developed above for the case of the double spherical pendulum. This example was studied by Marsden and Scheurle [1993a]. In that paper we examined the relative equilibria, their bifurcations and stability. In this section we are rather focusing on periodic and quasiperiodic orbits and their visualization, but the knowledge of the relative equilibria is a helpful guide.

\subsection{The Basic Equations and their Continuous Symmetry}

The double spherical pendulum system was shown in figure 1.1.1. Recall that we let the position vectors of each pendulum relative to their hinge points be denoted $\mathbf{q}_{1}$ and $\mathbf{q}_{2}$. These vectors are assumed to have fixed lengths $l_{1}$ and $l_{2}$ and the pendula masses are denoted $m_{1}$ and $m_{2}$. The 
configuration space is $Q=S_{l_{1}}^{2} \times S_{l_{2}}^{2}$, the product of spheres of radii $l_{1}$ and $l_{2}$ respectively. The Lagrangian is

$$
L\left(\mathbf{q}_{1}, \mathbf{q}_{2}, \dot{\mathbf{q}}_{1}, \dot{\mathbf{q}}_{2}\right)=\frac{1}{2} m_{1}\left\|\dot{\mathbf{q}}_{1}\right\|^{2}+\frac{1}{2} m_{2}\left\|\dot{\mathbf{q}}_{1}+\dot{\mathbf{q}}_{2}\right\|^{2}-m_{1} g \mathbf{q}_{1} \cdot \mathbf{k}-m_{2} g\left(\mathbf{q}_{1}+\mathbf{q}_{2}\right) \cdot \mathbf{k},
$$

where $\mathbf{k}$ is the unit vector along the $z$-axis. Since $\mathbf{q}_{1}+\mathbf{q}_{2}$ represents the position of the second mass relative to an inertial frame, (5.1.1) has the standard form of kinetic minus potential energy. We identify the velocity vectors $\dot{\mathbf{q}}_{1}$ and $\dot{\mathbf{q}}_{2}$ with vectors perpendicular to $\mathbf{q}_{1}$ and $\mathbf{q}_{2}$, respectively.

The conjugate momenta are

$$
\mathbf{p}_{1}=\frac{\partial L}{\partial \dot{\mathbf{q}}_{1}}=m_{1} \dot{\mathbf{q}}_{1}+m_{2}\left(\dot{\mathbf{q}}_{1}+\dot{\mathbf{q}}_{2}\right)
$$

and

$$
\mathbf{p}_{2}=\frac{\partial L}{\partial \dot{\mathbf{q}}_{2}}=\mathbf{m}_{2}\left(\dot{\mathbf{q}}_{1}+\dot{\mathbf{q}}_{2}\right)
$$

regarded as vectors in $\mathbb{R}^{3}$ that are only paired with vectors orthogonal to $\mathbf{q}_{1}$ and $\mathbf{q}_{2}$ respectively.

The Hamiltonian is therefore

$$
H\left(\mathbf{q}_{1}, \mathbf{q}_{2}, \mathbf{p}_{1}, \mathbf{p}_{2}\right)=\frac{1}{2 m_{1}}\left\|\mathbf{p}_{1}-\mathbf{p}_{2}\right\|^{2}+\frac{1}{2 m_{2}}\left\|\mathbf{p}_{2}\right\|^{2}+m_{1} g \mathbf{q}_{1} \cdot \mathbf{k}+m_{2} g\left(\mathbf{q}_{1}+\mathbf{q}_{2}\right) \cdot \mathbf{k} .
$$

The equations of motion are given by the Euler-Lagrange equations for $L$ on $T Q$ or, equivalently by Hamilton's equations for $H$ on $T^{*} Q$.

As for the symmetry group, let $G=S^{1}$ act on $Q$ by simultaneous rotation of the two pendula about the $z$-axis. If $R_{\theta}$ is the rotation by an angle $\theta$, the action is

$$
\left(\mathbf{q}_{1}, \mathbf{q}_{2}\right) \mapsto\left(R_{\theta} \mathbf{q}_{1}, R_{\theta} \mathbf{q}_{2}\right) .
$$

The infinitesimal generator corresponding to the rotation vector $\omega \mathbf{k}$, where $\omega \in \mathbb{R}$, is $\omega\left(\mathbf{k} \times \mathbf{q}_{1}, \mathbf{k} \times \mathbf{q}_{2}\right)$ and so the corresponding momentum map (conserved quantity) is the total angular momentum about the $z$-axis, given by

$$
\left\langle\mathbf{J}\left(\mathbf{q}_{1}, \mathbf{q}_{2}, \mathbf{p}_{1}, \mathbf{p}_{2}\right), \omega \mathbf{k}\right\rangle=\omega\left[\mathbf{p}_{1} \cdot\left(\mathbf{k} \times \mathbf{q}_{1}\right)+\mathbf{p}_{2} \cdot\left(\mathbf{k} \times \mathbf{q}_{2}\right)\right]=\omega \mathbf{k} \cdot\left[\mathbf{q}_{1} \times \mathbf{p}_{1}+\mathbf{q}_{2}+\mathbf{p}_{2}\right]
$$
i.e.,

$$
\mathbf{J}=\mathbf{k} \cdot\left[\mathbf{q}_{1} \times \mathbf{p}_{\mathbf{1}}+\mathbf{q}_{2} \times \mathbf{p}_{2}\right] .
$$

Note that from (5.1.2) and (5.1.3),

$$
\begin{aligned}
\mathbf{J} & =\mathbf{k} \cdot\left[m_{1} \mathbf{q}_{1} \times \dot{\mathbf{q}}_{1}+m_{2} \mathbf{q}_{1} \times\left(\dot{\mathbf{q}}_{1}+\dot{\mathbf{q}}_{2}\right)+m_{2} \mathbf{q}_{2} \times\left(\dot{\mathbf{q}}_{1}+\dot{\mathbf{q}}_{2}\right)\right] \\
& =\mathbf{k} \cdot\left[m_{1}\left(\mathbf{q}_{1} \times \dot{\mathbf{q}}_{1}\right)+m_{2}\left(\mathbf{q}_{1}+\mathbf{q}_{2}\right) \times\left(\dot{\mathbf{q}}_{1}+\dot{\mathbf{q}}_{2}\right)\right] .
\end{aligned}
$$

This system is one with a cyclic coordinate and so the mechanical connection and the locked inertia tensor may be constructed as explained in previous sections. In this case, the mechanical connection is

$$
\alpha\left(q_{1}, q_{2}, v_{1}, v_{2}\right)=\frac{\mathbf{k} \cdot\left[m_{1} q_{1} \times v_{1}+m_{2}\left(q_{1}+q_{2}\right) \times\left(v_{1}+v_{2}\right)\right]}{m_{1}\left\|q_{1}^{\perp}\right\|^{2}+m_{2}\left\|\left(q_{1}+q_{2}\right)^{\perp}\right\|^{2}},
$$

where $q^{\perp}$ denotes the horizontal projection of a vector $q$. The locked inertia tensor is the moment of inertia of the system about the $z$-axis regarded as a rigid structure. Thus,

$$
\mathbb{I}\left(\mathbf{q}_{1}, \mathbf{q}_{2}\right)=m_{1}\left\|\mathbf{q}_{1}^{\perp}\right\|^{2}+m_{2}\left\|\left(\mathbf{q}_{1}+\mathbf{q}_{2}\right)^{\perp}\right\|^{2}
$$

where $\left\|\mathbf{q}_{1}^{\perp}\right\|^{2}=\left\|\mathbf{q}_{1}\right\|^{2}-\left\|\mathbf{q}_{1} \cdot \mathbf{k}\right\|^{2}$ is the square length of the projection of $\mathbf{q}_{1}$ onto the $x y$-plane. Note that $\mathbb{I}$ is the moment of inertia of the system about the $\mathbf{k}$-axis and in this example, it is a scalar function on configuration space.

Here, the symplectically reduced space, reduced at a nonzero angular momentum value $\mu$, is $T^{*}\left(Q / S^{1}\right)$ which is 6 dimensional. It has a nontrivial magnetic term obtained by taking the differential of the $\mu$-component of (5.1.8). A convenient way of coordinatizing this reduced space is by coordinates $r_{1}=\left\|\mathbf{q}_{1}^{\perp}\right\|^{2}$ and $r_{2}=\left\|\mathbf{q}_{2}^{\perp}\right\|^{2}$ and the angle $\varphi$ between the vectors $\mathbf{q}_{1}^{\perp}$ and $\mathbf{q}_{2}^{\perp}$. 


\subsection{The Discrete Symmetries}

The double spherical pendulum has two basic discrete "symmetries", namely spatial reflection in vertical planes and time reversibility. We note that already in Marsden and Scheurle [1993], these discrete symmetries played an important role in the stability analysis of relative equilibria.

Obviously the simultaneous rotations of the two pendula together with spatial reflections in vertical planes represent an action of $O(2)$ on $Q$. Correspondingly, we obtain a lifted symplectic action on $T Q$ (one can work with either $T Q$ or $T^{*} Q$ as is convenient). The time reversibility acts on orbits in $T Q$ by $t \mapsto-t, \mathbf{q}_{i} \mapsto \mathbf{q}_{i}$ and $\dot{\mathbf{q}}_{i} \mapsto-\dot{\mathbf{q}}_{i}, i=1,2$. The map $\mathcal{R}:\left(\mathbf{q}_{i}, \dot{\mathbf{q}}_{i}\right) \mapsto\left(\mathbf{q}_{i},-\dot{\mathbf{q}}_{i}\right)$ generates an antisymplectic action of $\mathbb{Z}_{2}$ on $T Q$.

Note that $\mathcal{R}$ commutes with the $O(2)$-action. Thus, altogether, we get an action of the direct product $\mathbb{Z}_{2} \times O(2)$ on $T Q$.

Note also that $\mathcal{R}$ changes the sign of the momentum map $\mathbf{J}$ given in (5.1.5), as does any element in $O(2) \backslash S^{1}$. Thus, separately, these actions do not drop to the reduced phase space $\mathbf{J}^{-1}(\mu) / G_{\mu}$. However, it turns out that by combining the action by $\mathcal{R}$ with the action of transformation in $O(2) \backslash S^{1}$, one obtains a new action of $O(2)$ (with $S^{1}$ acting symplectically and transformation in $O(2) \backslash S^{1}$ acting antisymplectically) that preserves $\mathbf{J}$. Thus, it drops to a $\mathbb{Z}_{2}$-action on the symplectic reduced space. This is a reversible symmetry in the sense that it preserves the Lagrangian (or Hamiltonian), but its nontrivial element is antisymplectic.

To put the preceding observations in the framework of the previous sections, we make the following choice for $\Gamma$ as a subgroup of $\mathbb{Z}_{2} \times O(2)$ :

$$
\Gamma=\left\{(e, s) \mid s \in S^{1} \subset O(2)\right\} \cup\left\{(\sigma, s) \mid e \neq \sigma \in \mathbb{Z}_{2}, s \in O(2) \backslash S^{1}\right\}
$$

Obviously $\Gamma$ is isomorphic to $O(2)$, where we identify the normal subgroup $S^{1}$ with the set $\{(e, s) \mid$ $\left.s \in S^{1} \subset O(2)\right\}$. Thus, $\Sigma=\Gamma / S^{1}$, which is isomorphic to $\mathbb{Z}_{2}$, is our discrete symmetry group. It acts antisymplectically and clearly preserves $\mathbf{J}$, and so drops to an antisymplectic action on the symplectically reduced space.

We remark that this example shows that even for antisymplectic transformation, one can have $\mathbf{J}$ preserved in (4.3.4), since $-\sigma_{\mathfrak{g}^{*}}$ is the identity in this case.

There are many solutions of the reduced double spherical pendulum equation that have this type of reversible symmetry, that is, the corresponding orbits are $\mathbb{Z}_{2}$-symmetric. For example, consider the neighborhood of a (regular) point in the reduced phase space which corresponds to a relative equilibrium point of the pendulum problem. Take any values of the system parameters (the pendulum lengths and masses) such that the linearized vector field has pairs of simple purely imaginary eigenvalues which are not integer multiples of one another. Then by the Liapunov center theorem, there exists families of periodic orbits with frequencies approximately given by the imaginary parts of the eigenvalues. But by reversibility, for any solution, action by the nontrivial element of $\Sigma$ and time reversal produces another solution. Thus, by uniqueness of the Liapunov orbits within each energy surface, the orbits as sets are invariant under $\Sigma$. (For other systems, one can use the methods of Montaldi, Roberts and Stewart [1988, 1990] to find orbits with discrete symmetries). As we saw in the introduction, one can visualize this symmetry.

\section{Acknowledgments}

We thank Jeff Wendlandt for providing the computations shown in the introduction. We also thank Sergy Prishepionok and Isaac Kunin for useful discussions.

\section{References}

Bohr, H. A. [1947] Almost periodic functions, tr. by Harvey Cohn. New York, Chelsea Pub. Co., 1947; see also Fastperiodische Funktionen, Ergebnisse der Mathematik und ihrer Grenzgebiete, Springer-Verlag, 1974. 
Chossat, P. and M. Golubitsky [1988], Iterates of maps with symmetry, SIAM J. Appl. Math.19, $1259-1270$.

Guillemin, V. and S. Sternberg [1984] Symplectic Techniques in Physics. Cambridge University Press.

Kunin, I., F. Hussain, X. Zhou, and S.J. Prishepionok [1992]. Centroidal frames in dynamical systems I. Point vortices, Proc. Roy. Soc. Lon. A 439, 441-463.

Marsden, J.E. [1992], Lectures on Mechanics London Mathematical Society Lecture note series, 174, Cambridge University Press.

Marsden, J.E., R. Montgomery and T.S. Ratiu [1990] Reduction, symmetry, and phases in mechanics. Memoirs AMS 436.

Marsden, J.E. and T.S. Ratiu [1994] Symmetry and Mechanics. Texts in Applied Mathematics, 17, Springer-Verlag.

Marsden, J.E., T.S. Ratiu, and A. Weinstein [1984a] Semi-direct products and reduction in mechanics, Trans. Am. Math. Soc. 281, 147-177.

Marsden, J.E. and J. Scheurle [1993a] Lagrangian reduction and the double spherical pendulum, ZAMP 44, 17-43.

Marsden, J.E. and J. Scheurle [1993b] The reduced Euler-Lagrange equations, Fields Institute Comm. 1, 139-164.

Marsden, J.E., J. Scheurle and J. Wendlandt [1995] Visualization of orbits and and symmetries of the double spherical pendulum, (in preparation).

Marsden, J.E. and A. Weinstein [1974] Reduction of symplectic manifolds with symmetry, Rep. Math. Phys. 5, 121-130.

Melbourne, I., M. Dellnitz, and M. Golubitsky [1993] The structure of symmetric attractors. Arch. Rat. Mech. and An. 123, 75-98.

Montaldi, J.A., R.M. Roberts, and I.N. Stewart [1988] Periodic solutions near equilibria of symmetric Hamiltonian systems, Phil. Trans. R. Soc. Lond. A 325, 237-293.

Montaldi, J.A., R.M. Roberts, and I.N. Stewart [1990] Existence of nonlinear normal modes of symmetric Hamiltonian systems. Nonlinearity 3, 695-730, 731-772.

Newton, P.K. [1994] Hannay-Berry phase and the restricted three vortex problem. Physica D 79, $416-423$.

Patrick, G. W. [1994] Relative equilibria of hamiltonian systems with symmetry: linearization, smoothness and drift. Tech. Report, Univ. of Sask. TRE-94-3(2).

Scheurle, J. [1981] Verzweigung Quasiperiodischer Lösungen bei Reversiblen Dynamishen Systemen. Habilitationsschrift, Universität Stuttgart. 